\title{
Cáncer y depresión: una revisión
}

\section{Cancer and depression: A review}

\author{
Rosario Fátima Diz Ramírez" Alberto Garza de la Cruz², \\ Elivier Kristina Olivas Verduzco3, José Guadalupe Montes Esqueda ${ }^{4}$ \\ y Guadalupe Selene Fernández Luciano
}

Citación: Diz R., R.F, Garza C., A., Olivas V., E.K., Montes E., J.G. y Fernández L., G.S. (2019). Cáncer y depresión: una revisión. Psicología y Salud, 29(1), 115-124.

\section{RESUMEN}

\begin{abstract}
El objetivo del presente escrito es mostrar el estado actual de la cuestión relativa al vínculo entre cáncer y depresión. Para llevarlo a cabo, se hizo una búsqueda limitada a artículos arbitrados publicados de 2012 a 2017 en las bases de datos EBSCO, RedAlyC y Google Académico mediante la unión de las palabras "cáncer" y "depresión", sin filtro alguno en cuanto al idioma, por lo que se tomaron en cuenta artículos en español e inglés, principalmente. Se obtuvo un total de 25 artículos viables para los fines de esta investigación, los cuales se analizaron, extractaron y clasificaron en categorías. La información aquí presentada se estructuró en forma de ensayo y en secciones específicas a fin de dar orden y coherencia a las aportaciones de los distintos autores. Así, dichas secciones abordan la definición del término "cáncer" y la prevalencia de esta enfermedad en México y en el mundo; algunos datos hallados en diversas investigaciones hechas acerca de la comorbilidad entre cáncer y depresión; las características clínicas de la depresión, sus causas y consecuencias en pacientes oncológicos; la evaluación de la depresión en dichos pacientes y, por último, los tratamientos para los síntomas depresivos, divididos en dos modos de abordarlos: el médico-biológico y el psicológico.
\end{abstract}

Palabras clave: Cáncer, Depresión; Tratamiento; Bases de datos; Pacientes oncológicos.

\section{SUMMARY}

The aim of this article is to show the current state of the question concerning the link between cancer and depression, through a search of articles published from 2012 to 2017 in EBSCO, RedALYC and Academic Google data bases using the union of the terms "cancer" and "depression", without idiomatic filters. Twenty five articles were found, analyzed, summarized and categorized. The information was structured as an essay divided in specific sections to give order and coherence to the contributions of the reviewed studies. Thus, such sections deal with the definition of the term "cancer" and the prevalence of this illness in Mexico and abroad. Some findings on cancer and depression revealed the clinical characteristics, causes and consequences of depression in oncological patients, the ways they were assessed and treatments provided to depressive symptoms, divided in two classes: medical-biological and psychological.

Key words: Cancer; Depression; Treatment; Data bases; Oncological patients.

\footnotetext{
${ }^{1}$ Universidad del Valle de Atemajac, Plantel Zamora, Valladolid 64, Fracc, Valencia 2a secc, 59610 Zamora, Mich., México, tel, (351)517-74-41, correo electrónico: fatimadiz13@hotmail,com, Artículo recibido el 26 de octubre de 2017 y aceptado el 2 de febrero de 2018.

${ }^{2}$ Hospital Regional de Reynosa PEMEX, Sierra Nevada 1342, Col, Fuentes, Sección Lomas, 88730 Reinosa, Tamps., México, tel, (899)148-73-10, correo electrónico: maestriareynosa@gmail.com.

${ }^{3}$ Universidad Autónoma de Baja California, Lago Pátzcuaro 790-B, Valle Dorado, 22890 Ensenada, B.C., México, correo electrónico: psic.elivierk@gmail.com.

${ }^{4}$ Centro de Psicoterapia Cognitiva, Vicente Guerrero 123, Col Miguel de Madrid, 46400 Tequila, Jal., México, correo electrónico: jgme_tequila@hotmail.com.

${ }^{5}$ Fiscalía General del Estado de Tabasco, Fracc, Estrellas de Buena Vista, C, Lyra, Manzana 42, Lote 3, 86250 Villahermosa, Tab., México, correo electrónico: selenef1982@gmail.com.
} 


\section{Cáncer: definición y prevalencia}

$\mathrm{E}$ 1 cáncer es la segunda causa de muerte en el mundo, y se considera la depresión como la enfermedad mental de este siglo, por lo que la ocurrencia de ambas enfermedades en una sola persona puede convertirse en una combinación deletérea, pues es posible que quien padece cáncer desarrolle síntomas depresivos en algún momento de la enfermedad, en tanto que la depresión aumenta la posibilidad de desarrollar enfermedades crónicas. Es de suma importancia detectar procesos depresivos de importancia clínica en pacientes oncológicos a fin de minimizar en ellos las consecuencias negativas de dicha combinación. El trabajo multidisciplinario se hace necesario en el proceso de diagnóstico de los procesos depresivos y en su tratamiento. Si bien existen innumerables investigaciones que abordan adecuadamente al paciente oncológico deprimido desde un punto de vista psicológico, aún falta desarrollar sistemas holísticos, sistemáticos y generalizados que atiendan este problema mundial.

El cáncer se define como "una alteración caracterizada por el crecimiento tisular patológico, el cual tiene su origen en una proliferación persistente y prolongada de células anormales que desemboca en una patología, determinada ésta por la invasión y destrucción de los tejidos del cuerpo" (Guerrero, Prepo y Loyo, 2016, p. 299).

La Organización Mundial de la Salud (OMS) (2017a) afirma que «una característica definitoria del cáncer es la multiplicación rápida de células anormales que se extienden más allá de sus límites habituales y que pueden invadir partes adyacentes del cuerpo o propagarse a otros órganos, un proceso que se denomina "metástasis". Las metástasis son la principal causa de muerte por cáncer» $(\S 10)$.

Otra definición se encuentra en Ortiz et al. (2014), quienes indican que "el cáncer es una enfermedad provocada por la proliferación anormal de células mediante un proceso denominado carcinogénesis, es decir, la capacidad que adquieren algunas células para multiplicarse incontroladamente e invadir otros órganos" (p. 78).

Según cifras de la OMS (2017b), “el cáncer es la segunda causa de muerte en el mundo; en 2015 ocasionó 8.8 millones de defunciones. Casi una de cada seis defunciones en el mundo se debe a esta enfermedad" ( $(3)$. Por otro lado, la misma organización enlista los cinco tipos de cáncer que causan un mayor número de fallecimientos en el mundo: pulmonar, hepático, colorrectal, gástrico y mamario.

El perfil oncológico de México, según la OMS (2014), indica que el total de fallecimientos causados por cáncer en los hombres fue de 33,900, siendo el de próstata el tipo de cáncer más frecuente, seguido por el pulmonar, el colorrectal, el gástrico y la leucemia. En el caso de las mujeres, se registraron 38,000 muertes, sobre todo por cáncer de mama, pero también cervicouterino, colorrectal, gástrico y hepático.

Además, el cáncer, "es considerado un grave problema de salud pública debido a las repercusiones biológicas, psicológicas y sociales para el paciente, así como por los altos costos económicos y de recursos humanos que representa para el Estado" (Guerrero et al., 2016, p. 298).

\section{Comorbilidad entre cáncer y depresión}

Berenzon, Lara, Robles y Medina-Mora (2013) señalan que la relación entre las enfermedades mentales y físicas es muy estrecha. A la mayoría de las personas les resulta difícil aprender a sobrellevar y vivir con una dolencia crónica de larga duración, como la diabetes o el cáncer, por lo que no es sorprendente que la depresión sea la complicación más común asociada con este tipo condiciones.

Del mismo modo, las personas que sufren depresión tienen mayores probabilidades de padecer enfermedades cardiovasculares y respiratorias, diabetes y cáncer, condiciones crónicas todas ellas responsables de más de $60 \%$ de las muertes en el mundo. Según los citados autores, la relación entre el cáncer y la depresión es un camino de doble vía, pues quien padece cáncer puede desarrollar síntomas depresivos en algún momento de la enfermedad, y la depresión aumenta la probabilidad de desarrollar cáncer.

Moreno, Krikorian y Palacio (2015) afirman que el cáncer puede ocasionar un grave impacto en las áreas física, psicológica, social y espiritual de una persona, y que su diagnóstico favorece el desarrollo de problemas para ella, su familia y su comunidad, en cualquier fase de su trayecto [cf. Bultz y Carlson, 2006; Bultz y Johansen, 2011]. 
"Una vez que la enfermedad es diagnosticada, ingresa a la cotidianidad de la persona y de su entorno y afecta su seguridad y confianza puesto que introduce cambios para los cuales el enfermo no estaba preparado" (p. 2).

Park y Rosenstein (2015) hallan que la prevalencia de síntomas depresivos en los pacientes con cáncer puede ir de 16 a 42\%, y estiman que "en todos los pacientes con cáncer, $20 \%$ puede tener depresión menor o distimia" (p. 3). A su vez, Bernal y Muñoz (2017) refieren que la cantidad de pacientes con cáncer afectados por depresión va de 15 a $25 \%$; además, afirman que "el estilo de afrontamiento depresivo se relaciona con un menor tiempo de supervivencia [...] alarga el tiempo de hospitalización, empeora la adherencia terapéutica y disminuye la calidad de vida" (p. 2).

"Varios estudios -apuntan Guerrero et al., 2016)- han confirmado la presencia de depresión y ansiedad durante el proceso patológico de cáncer, por lo que la evolución, pronóstico y tratamiento del cáncer se ve afectada por [dichos] trastornos" (p. 299). De acuerdo con Costa y Ballester (2010), parece haber una mayor prevalencia de síntomas ansiosos que depresivos en pacientes oncológicos hospitalizados, los cuales tienden a disminuir durante la enfermedad, en tanto que los síntomas depresivos se mantienen o aumentan al avanzar la enfermedad o en el postratamiento (Moreno et al., 2015).

Para concluir este apartado, conviene rescatar lo que Hernández et al. (2012) afirman: «El malestar emocional se está convirtiendo en un elemento capital para valorar la salud y el bienestar de los pacientes con cáncer. Para los clínicos, es el "sexto signo vital", tan principal como la temperatura, la respiración, el ritmo cardiaco, la presión sanguínea o el dolor» (p. 236).

\section{Características clínicas de la depresión}

Con el fin de no confundir los sentimientos naturales de tristeza que pueden surgir en las personas que reciben un diagnóstico como el cáncer, se vio que era necesario definir claramente las características de la depresión, las cuales se sintetizan a continuación.

La depresión es un síndrome clínico, generalmente clasificado como un trastorno mental; específicamente en los manuales de diagnóstico, se la clasifica como un trastorno del humor o del afecto. Para Diz (2017), los síntomas generales de la depresión se pueden clasificar en cuatro grandes áreas a fin de tener una visión más organizada del síndrome, entre los que se hallan, a saber:

- Síntomas afectivos: “Ánimo deprimido o triste, sensación de vacío que puede acompañarse de aplanamiento afectivo, infelicidad, incapacidad para disfrutar de cosas que anteriormente se apreciaban, sentimientos de culpa, inutilidad o inferioridad; sensaciones de impotencia y altos niveles de ansiedad" (pp. 29-30). En el caso de los pacientes con cáncer, es altamente probable que los síntomas afectivos se consideren "normales" por parte del propio paciente y de quienes le rodean; sin embargo, su persistencia puede ser el indicio de una depresión clínica.

- Síntomas cognitivos: "Problemas en la atención, concentración, memoria, capacidad de decisión y alteración del juicio, ya que [el paciente] tiene creencias fatalistas (perspectiva sombría de sí mismo y del futuro) y pensamientos de muerte o ideas suicidas" (p. 30). Galindo, Rojas, Meneses, Aguilar y Alvarado (2016) afirman que "la depresión en el paciente con cáncer suele asociarse a pensamientos negativos intrusivos relacionados a la enfermedad y la muerte, que a su vez contribuyen a que se mantengan estrategias de afrontamiento poco adaptativas y una visión negativa del futuro" (p. 44).

- Síntomas conductuales: "Elentecimiento o agitación psicomotriz, reducción de la energía y por consecuencia reducción del nivel de actividad; puede presentarse conducta suicida o autodestructiva, entre otras" (Diz, 2017, p. 30).

- Síntomas somáticos: "Desgano y fatiga, alteraciones en el apetito (incremento o decremento), alteraciones en el peso (generalmente pérdida), alteraciones en el sueño (insomnio o hipersomnia) y, por último, afectación de la conducta sexual (pérdida de la líbido)" (Diz, 2017, p. 30). En el paciente con cáncer, los síntomas conductuales y los somáticos pueden explicarse no solamente por la depresión, sino también -y principalmentepor la enfermedad física que lo aqueja. 
La duración de los síntomas depresivos determinará si se diagnostica un episodio depresivo mayor, en el que se requiere que la persona manifieste los síntomas depresivos por al menos dos semanas, o un trastorno distímico, cuya duración es por lo regular de dos años o más.

Los niveles de gravedad de la depresión pueden distinguirse si se analizan la intensidad, duración y frecuencia de los síntomas presentes. Por ejemplo, "el trastorno depresivo leve es frecuente y se caracteriza por humor y conducta alterados, dificultades con el sueño, pesimismo, ansiedad y falta de energía. En el trastorno depresivo moderado, los pacientes manifiestan un descenso del humor, ansiedad, irritabilidad y agitación, así como falta de interés, disfrute y concentración y mala memoria [...] En el trastorno depresivo grave los síntomas [...] aumentan en intensidad y aparecen [otros] nuevos, como delirios y alucinaciones" (Di Natale et al., 2012, p. 21).

La depresión en los pacientes con cáncer, según Fuchs, Bejarano, Álvarez, Godoy y Fernández (2013), suele ser breve al principio y después durar varios días o semanas; "puede incluir sentimientos de incredulidad, rechazo o desesperación, o bien comenzar con un período disfórico, en el cual se pueden experimentar trastornos del sueño y del apetito, ansiedad, cavilaciones y temor al futuro" (p. 191).

Hernández y Cruzado (2013) resumen la situación de los síntomas depresivos en los pacientes oncológicos de la siguiente manera: "Pueden presentar temores, preocupaciones, aprensión, sentimientos de indefensión, pesadillas e insomnio, disfunciones y problemas sexuales, y secuelas neuropsicológicas a corto y largo plazo que pueden alcanzar al 43\% de los pacientes según el tipo de tumor y de radioterapia" (p. 2).

Así, como síndrome clínico, la depresión puede influir en el curso de las enfermedades físicas, ya sea porque el paciente tiene actitudes que no apoyan los procesos de tratamiento, o bien porque en sí misma afecta procesos vitales de importancia, como el sistema inmune. Como afirman Sánchez, Figueroa, Cacho y Robles (2016), "los factores psicosociales que se encuentran presentes en la vida cotidiana provocan estados de ansiedad, depresión y estrés, causando un desequilibrio en las funciones de los sistemas autonómico, endócrino e inmune que a su vez puede originar el desarrollo o progresión tumoral" (p. 151). Por esta razón, De Paula, Sonobe, Nicolussi, Zago y Sawada (2012) apuntan la importancia de que los profesionales de la salud "detecten oportunamente los síntomas de depresión para mejorar la calidad de vida en estos pacientes" (p. 6).

\section{Causas de la depresión en pacientes oncológicos}

La persona que ha sido diagnosticada con cáncer "es un individuo vulnerable ante la depresión generada por la propia enfermedad, pero también por otros factores, como su contexto y desarrollo; por consiguiente, tales variables influyen en el éxito de su tratamiento (Velásquez y Zuluaga, 2015).

Las causas de la depresión en pacientes oncológicos son muy variadas, y entre ellas se encuentran la edad (de hecho, en ocasiones es más frecuente en pacientes jóvenes). El género es otra causa, aunque no es común en todos los estudios, y también el estadio en que se halla la enfermedad, debido tal vez a la mayor limitación o la incapacidad física que trae consigo la misma; igualmente destaca la presencia de síntomas físicos resultantes de los tratamientos; el tipo de enfermedad neoplásica (los pacientes más deprimidos son los que sufren cáncer pancreático, orofaríngeo y del sistema nervioso central); los antecedentes psicológicos del paciente, los trastornos previos que haya experimentado, el escaso apoyo social percibido y los factores de riesgo biopsicosocial (Hernández et al., 2012).

Según Pousa et al. (2015), los factores de riesgo con mayor peso para el desarrollo de depresión en pacientes con cáncer son los siguientes: a) el tipo de cáncer (cánceres que presentan síndromes paraneoplásicos y cánceres con liberación de citoquinas); b) el estadio del cáncer (se observa una mayor presencia de trastorno mental en los pacientes más sintomáticos); c) el tratamiento oncológico en curso; d) los antecedentes de depresión (tener un diagnóstico previo de depresión es el mayor factor de riesgo para desarrollar este trastorno en una enfermedad oncológica); e) el dolor (sobre todo el dolor crónico persistente o no bien controlado); f) una red social limitada; $g$ ) un estado funcional pobre, y $h$ ) la edad (los pacientes con menos edad son más propensos a sufrir depresión). 
Es frecuente, además, encontrar pacientes oncológicos con ideación suicida, provocada por las situaciones específicas de cada caso, como pueden ser un estadio avanzado y con mal pronóstico de la enfermedad; el tipo y localización del tumor, mayor en el cáncer de cabeza y cuello; la presencia de un trastorno de depresión, o la desesperanza, la falta de control y el sentimiento de desamparo; el incremento de síntomas físicos o su cronicidad, el delirium y los estados confusionales; los antecedentes psicopatológicos, y la historia previa de intento de suicidio (Hernández et al., 2012).

\section{Consecuencias de la depresión en pacientes oncológicos}

La asociación entre cáncer y depresión "no solo produce sufrimiento, sino también una peor calidad de vida. Se ha visto que reduce el cumplimiento del tratamiento anticancerígeno, puede llevar al suicidio, es una carga psicológica para la familia y prolonga el tiempo de hospitalización" (Bernal y Muñoz, 2017, p. 2). La depresión, además, disminuye la habilidad del paciente para afrontar la enfermedad y agudiza la cantidad y la intensidad de los síntomas físicos (Colleoni et al., 2000; Prieto et al., 2002). Por último, la presencia de depresión en el curso de las enfermedades oncológicas reduce la adherencia terapéutica e incrementa la mortalidad y la morbilidad (Teng, De Castro y Navas, 2005). En conclusión, cáncer y depresión forman una combinación mortal. En una revisión sistemática hecha por Barber et al. (2016) de pacientes con cánceres de mama, pulmón, cerebro, piel y sangre, se encontró una mortalidad 25\% mayor en pacientes con síntomas depresivos, y 39\% mayor en pacientes con depresión mayor o menor, incluso cuando se controlan los pronósticos clínicos conocidos de la supervivencia.

\section{Evaluación de la depresión en pacientes oncológicos}

La detección oportuna de la depresión en pacientes oncológicos reviste suma importancia, ya que hacer un diagnóstico confiable conlleva un mejor manejo no solo de la sintomatología causada por la enfermedad oncológica, sino también una adecuada intervención en lo que se refiere a los síntomas de depresión. Según Landa et al. (2014), el clínico debe decidir el instrumento a utilizar para evaluar la depresión, evaluación que puede ser hecha por medio de preguntas o a través de cuestionarios. Estos mismos autores afirman: "Las evaluaciones de una o dos preguntas son utilizadas con mayor frecuencia, tanto en la práctica clínica como en la investigación en la población paliativa. Se consideran útiles para recabar información oportuna sobre el estado de ánimo del paciente. Más que para establecer un diagnóstico, se emplean como una herramienta de tamizaje [...] Por otra parte, los cuestionarios de evaluación son instrumentos con mejores propiedades psicométricas debido a la cantidad de reactivos, que varían entre 5 a 20 por instrumento, y suelen evaluar un espectro más amplio de alteración emocional" (p. 416).

Para una detección de la depresión más acertada, se recomienda una evaluación en la que se combinen algunas preguntas de tipo exploratorio al principio; si se detectan síntomas significativos, es deseable aplicar alguna prueba especializada que, además, haya sido estandarizada previamente. Al respecto, Galindo et al. (2015) llevaron a cabo un estudio de la Escala Hospitalaria de Ansiedad y Depresión (HADS), prueba desarrollada y publicada en 1983 por Zigmond y Snaith. Esta herramienta consta de 14 ítems, en un formato de autoaplicación; incluye dimensiones cognitivas y afectivas y omite aspectos somáticos tales como el insomnio, la fatiga, la pérdida de apetito y otros para evitar atribuirlos a la depresión y no a la enfermedad. En dicho estudio, los autores encontraron que la escala original había sido adaptada y validada en diversas poblaciones y culturas, mostrando siempre una sensibilidad y especificidad adecuadas para discriminar ansiedad y depresión. Afirman los autores del estudio: "La ventaja de la HADS sobre otros instrumentos que evalúan sintomatología ansiosa y depresiva es que no incluye síntomas somáticos que pueden explicarse por el cáncer y su tratamiento [...] Al ser un instrumento breve, fácil de aplicar y confiable para la práctica clínica y la investigación en población oncológica, resulta relevante para determinar la magnitud de la problemática, prevención e implementación de acciones para el tratamiento" (pp. 254-257).

Por otro lado, Galindo et al. (2016) investigaron las propiedades psicométricas del Inventa- 
rio de Depresión de Beck II (BDI-II), estructurado por Beck, Steer y Brown (1996), en pacientes con cáncer. El BDI-II es un instrumento de autoinforme compuesto por 21 ítems elaborados para evaluar los síntomas correspondientes a los criterios diagnósticos de los trastornos depresivos del Manual Diagnóstico y Estadístico de los Trastornos Mentales (DSM-V) (American Psychatric Association, 2016). En el citado estudio, se llegó a las siguientes conclusiones: "El BDI-II ha demostrado ser un instrumento válido y confiable para detectar sintomatología depresiva [...] mostró características psicométricas adecuadas en su aplicación a pacientes con cáncer en población mexicana. La relevancia de los resultados obtenidos radica en que se trata de una población que puede requerir atención oportuna en su salud mental a lo largo del tratamiento oncológico" (p. 43).

Las investigaciones relacionadas con la evaluación de la depresión en pacientes con cáncer buscan encontrar instrumentos confiables y válidos que permitan detectar la presencia de síntomas depresivos con niveles clínicamente significativos, e intervenir oportunamente en la atención integral del paciente oncológico para lograr un mejor pronóstico y un curso de la enfermedad más esperanzador.

\section{Tratamiento de síntomas depresivos en pacientes oncológicos}

E1 tratamiento de los síntomas depresivos se ha abordado típicamente desde muy diversos ángulos, los que a continuación se presentan agrupados en categorías para su mejor comprensión.

\section{- Abordaje físico}

El principal modo de abordar la depresión desde el área somática se encuentra en el manejo farmacológico. Bernal y Muñoz (2017) señalan que "se ha encontrado que los fármacos inhibidores de la recaptación de serotonina son eficaces para el tratamiento de la depresión en pacientes con cáncer. La paroxetina es una de las más eficaces. Los antidepresivos de perfil dual también han demostrado eficacia por su rapidez de acción y su utilidad en síntomas colaterales, como los síntomas vasomotores y los dolores. La mirtazapina es de gran uti- lidad en síntomas como el dolor, náuseas, insomnio y ansiedad (p. 249).

Los mismos autores indican que quien debe prescribir este tipo de tratamientos es el médico oncólogo en colaboración con el médico psiquiatra, ya que elegir la medicación apropiada debe hacerse sobre la base del perfil síntomático del paciente, sus beneficios, la tolerabilidad y el riesgo-beneficio con el propósito de diseñar una estrategia para cada paciente" (pp. 249-250). El trabajo multidisciplinario se hace necesario ya que, como lo reportan Pousa et al. (2015), "con pacientes oncológicos sometidos a varias quimioterapias, es importante tener precaución con las interacciones farmacológicas. Antidepresivos como la fluoxetina, la sertralina, la paroxetina y la fluvoxamina inhiben la transformación de agentes antineoplásicos a través del CYP450 3A4, lo que produce una disminución de su eficacia o mayor toxicidad de los mismos. Otros fármacos como el citalopram, el escitalopram, la venlafaxina y la mirtazapina son inhibidores débiles, lo que los convierte en una elección más segura (p. 169).

Algunas recomendaciones que complementan la intervención farmacológica las formulan Olivan et al. (2016): "Es necesario que el médico familiar pueda establecer un tratamiento específico para los síntomas de la depresión, como el estado de ánimo bajo, la anhedonia, las dificultades para conciliar el sueño o la falta de un descanso reparador con un medicamento farmacológico específico, técnicas de modificación de conducta e higiene del sueño, entre otros (p. 63).

\section{- Abordaje psicológico}

Los resultados del estudio realizado por Aguilar et al. (2014), muestran que se requiere una guía psicológica oportuna y la terapia apropiada desde el momento en que se diagnostica a las personas con cáncer. Es decir, no debe esperarse a que el paciente diagnosticado con cáncer manifieste un trastorno psicológico, toda vez que la intervención en los niveles iniciales (prevención primaria) contribuye a prevenir la ocurrencia de cuadros psicopatológicos clínicos posteriores, como la depresión.

Cuando la depresión ya está presente e incluso ha sido diagnosticada, será necesario llevar a cabo una intervención psicológica que minimice 
su impacto en la enfermedad física (prevención secundaria), para así prevenir su exacerbación.

En este apartado se exponen los sistemas de abordaje psicológico más citados en la literatura especializada, clasificándolos en dos modalidades: la individual y la familiar/social.

\section{- Abordaje psicológico individual}

Una de las psicoterapias individuales más citadas en la literatura científica actual, la cual ha mostrado una alta eficiencia para abordar la depresión, es la terapia cognitivo-conductual de Beck. Al decir de Beltrán, Freyre y Hernández (2012), "se basa en un modelo de vulnerabilidad al estrés, en el que se activan ciertos esquemas de pensamiento distorsionado que contribuyen a la manera negativa en que el individuo percibe, codifica e interpreta la información sobre sí mismo, sobre el mundo y sobre el futuro, lo que inicia y mantiene los síntomas depresivos" (p. 6).

El objetivo de esta terapia es que el paciente reestructure sus creencias sobre sí mismo, el mundo y el futuro de un modo más realista, que lo conduzca a un modo más sano de afrontar la vida en general.

Por otro lado, estrategias como el mindfulness, o atención plena, han adquirido gran relevancia en los últimos años; dicha estrategia, derivada de las prácticas meditativas del budismo Zen, consiste, en palabras de Kabat-Zinn, en "prestar atención de un modo particular: deliberadamente, en el momento presente y sin juzgar". Es decir, ser plenamente conscientes de lo que ocurre en el momento presente, sin filtros ni prejuicios (Pousa et al., 2015, p. 4).

Otras estrategias, como la relajación y otras terapias basadas en la imaginación, se han utilizado para realizar intervenciones exitosas, consignadas en numerosas publicaciones. Entre sus efectos se encuentran la reducción del impacto sobre el cuerpo del estrés asociado al desencadenamiento del cáncer y al miedo a la enfermedad, la activación del sistema inmunológico contra la enfermedad mediante la instauración de creencias positivas, el aumento de la motivación para el cambio del estilo de vida, el fortalecimiento del "deseo de vivir", el afrontamiento de la desesperanza y la evaluación y modificación de las creen- cias del enfermo respecto a la enfermedad (Pousa et al., 2015).

Guerrero et al. (2016) afirman que uno de los mecanismos usados para contrarrestar los efectos negativos de la ansiedad y la depresión en pacientes oncológicos podría ser la autotrascendencia (espiritualidad), definida como "la reducción de las creencias personales y conductas que expresan una cosmovisión individual en un sentido de relación con una dimensión trascendente, que adquiere gran significación en circunstancias diversas de la enfermedad, explicadas como momentos cruciales en la vida del individuo, pudiendo ayudar a aceptar la enfermedad y lograr que otras personas acepten dicha condición de salud [...] La autotrascendencia influye sobre la salud y el bienestar, siendo a su vez promotora de comportamientos saludables que representan un aporte significativo a la salud de los pacientes con cáncer" (pp. 299-300).

En múltiples investigaciones se sigue trabajando para estructurar métodos específicos para el manejo de la depresión en pacientes oncológicos; mientras tanto, es necesario que los profesionales de la salud mental intervengan con los métodos ya conocidos para el tratamiento de la depresión clásica, y hagan un seguimiento puntual del curso de los síntomas anímicos y físicos en beneficio del propio paciente y de su familia.

\section{- Abordaje psicológico familiar/social}

Cuando aparece una enfermedad como el cáncer, la familia funge como la mayor fuente de apoyo social, personal, afectivo y económico; es por ello que el profesional de la salud mental debe abordar las posibles vicisitudes que surgen en el grupo de apoyo familiar o social del paciente oncológico.

Por ejemplo, los conflictos intrafamiliares y la culpa pueden empeorar el curso de la enfermedad, la cercanía y la unidad, mientras que las relaciones de apoyo mutuo y las destrezas de la familia en el cuidado del enfermo son importantes factores de protección (Fuchset al., 2013).

El abordaje psicológico de la familia y de las personas que apoyan al paciente oncológico se ha realizado clásicamente a través de las intervenciones en grupos terapéuticos o grupos de apoyo; sin embargo, el profesional de la salud mental de- 
berá evaluar además, si se requiere, la intervención individual con algún familiar o un amigo en particular.

Por otro lado, el personal médico y de enfermería necesita recibir entrenamiento para realizar un adecuado manejo del paciente oncológico deprimido, lo que está llevando a psicólogos y psiquiatras a invertir un tiempo considerable en la formación de equipos sanitarios en psicopatología, y asimismo en el desarrollo de instrumentos de detección adecuados a la realidad asistencial de los pacientes y que les ayuden a detectar los problemas emocionales con los objetivos de brindarles orientación y ayuda psicológica desde las fases más tempranas del tratamiento médico, reducir los costes de cuidados sanitarios y programar la atención sanitaria para los pacientes con un malestar psicológico considerable sin necesidad de largas entrevistas diagnósticas o extensos cuestionarios (Hernández et al., 2012).

Por último, es necesario recordar que la atención integral debe llevarla a cabo todo aquel profesional que participe en la atención de cualquier persona enferma; el médico o enfermero que limite su intervención a los meros aspectos físicos, así como el psicólogo que la restrinja a los psicológicos, estarán realizando un abordaje parcial que puede ser dañino para la salud de sus pacientes. En el caso específico de los pacientes con cáncer, el descuido de alguna de estas dimensiones físicas, psicológicas y sociales puede repercutir en el curso de la enfermedad y desembocar en un pronóstico desfavorable.

\section{CONCLUSIONES}

Las principales conclusiones que se pueden extraer del análisis de las investigaciones actuales sobre cáncer y depresión son las siguientes:

El cáncer es una enfermedad que se ha convertido en un grave problema de salud pública, con importantes consecuencias individuales, familiares y sociales.
La comorbilidad entre cáncer y depresión es elevada, por lo que los profesionales de la salud deben evaluar si un paciente cursa ambas enfermedades a fin de ofrecerle un tratamiento oportuno e integral.

Los síntomas de la depresión en el paciente oncológico son múltiples y muy variados y están conectadas al tipo de cáncer, al estadio en el que se encuentra la enfermedad, al tipo de tratamiento recibido, a la edad del paciente, a la presencia o no de dolor y a las redes sociales de apoyo de que aquél dispone, entre otros factores, por lo que se requiere una capacitación especial para que el profesional de la salud identifique correctamente esos signos y síntomas.

Las consecuencias de la depresión en pacientes oncológicos también son múltiples, entre las que se encuentran el daño a su calidad de vida y la disminución de su adhesión al tratamiento oncológico; al parecer, muestran un mayor índice de mortalidad los pacientes que padecen ambos trastornos.

El abordaje de los síntomas depresivos en dichos pacientes ha incluido tratamientos medicamentosos cuyos resultados son variables, pero también psicológicos, tanto en el plano individual -como es el caso de la TCC de Beck, el mindfulness y otras-, como en el familiar y social, donde se trabaja con las redes de apoyo para que se manejen adecuadamente los procesos físicos y psicológicos por los que atraviesan estos enfermos.

Por último, es necesario hacer énfasis en que la dicotomía cuerpo-mente es un tema actual, y se le puede percibir claramente al momento en que se analiza la literatura sobre cáncer y depresión, ya que esta da cuenta de que estos dos padecimientos se encuentran íntimamente vinculados; sin embargo, aún no existen sistemas integrales de intervención para el paciente oncológico que sean además sistemáticos y generalizados, en los que se aborde la enfermedad de un modo holístico, lo que traería consigo un sustancial beneficio al paciente. 


\section{REFERENCIAS}

Aguilar C., M.J., Mur V., N., Neri S., M., Pimentel R., M.L., García R., A. y Gómez V., E. (2014). Breast cancer and body image as a prognostic factor of depression: a case study in México City. Nutrición Hospitalaria, 31(1), 371-379. doi: 10.3305/ nh.2015.31.1.7863.

American Psychatric Association (2016). Manual Diagnóstico y Estadístico de los Trastornos Mentales (5 ed.). Ciudad de México: Editorial Médica Panamericana.

Barber, B., Dergousoff, J., Slater, L., Harris, J., O’Connell, D., El-Hakim, H., Biron, V., Mitchell, N. y Seikaly, H. (2016). Depression and survival in patients with head and neck cancer: a systematic review. JAMA Otolaryngology: Head \& Neck Surgery, 142(3), 284-288. doi: 10.1001/jamaoto.2015.3171.

Beck, A.T., Steer, R.A. y Brown, G.K. (1996). Manual for the Beck Depression Inventory-II. San Antonio, TX: Psychological Corporation.

Beltrán M., C., Freyre M., Á. y Hernández G., L. (2012). El Inventario de Depresión de Beck: su validez en población adolescente. Terapia Psicológica, 30(1), 5-13.

Berenzon, S., Lara, M.A., Robles, R. y Medina-Mora, M.E. (2013). Depresión: estado del conocimiento y la necesidad de políticas públicas y planes de acción en México. Salud Pública de México, 55(1), 74-80.

Bernal G., P. y Muñoz A., M. (2017). Tratamiento farmacológico de la depresión en cáncer. Psicooncología, 13(2-3), 249-270. doi: 10.5209/PSIC.54435.

Bultz, B.D. y Carlson, L.E. (2006). Emotional distress: the sixth vital sign — future directions in cancer care. Psycho-Oncology, 15(2), 93-95.

Bultz, B. y Johansen, C. (2011). Screening for distress, the 6th Vital Sign: where are we, and where are we going? Psycho-Oncology, 20, 569-571.

Colleoni, M., Mandala, M., Peruzzotti, G., Robertson, C., Bredart, A. y Goldhirsch, A. (2000). Depression and degree of acceptance of adjuvant cytotoxic drugs. Lancet, 356, 1326-1327.

De Paula, J.M., Sonobe, H.M., Nicolussi, A.C., Zago, M.F. y Sawada, N.O. (2012). Symptoms of depression in patients with cancer of the head and neck undergoing radiotherapy treatment: a prospective study. Revista Latino-Americana Enfermagem, 20(2), 362-368.

Di Natale G., Renzo G., Espinal M., Sucre A., I.T., Dávila S., J.L, Hanssen H., D.A. y Lechtig B., A. (2012). Grados de depresión en padres de niños con cáncer. Revista Venezolana de Oncología, 24(1) 19-26.

Diz R., R.F. (2017). Características de la depresión en el siglo XXI. Revista de la Universidad del Valle de Atemajac, 36(87), 22-31.

Fuchs T., V., Bejarano, M., Álvarez A., K., Godoy, M. y Fernández K., N.C. (2013). Efecto de la presencia de los familiares sobre la depresión en mujeres hospitalizadas con cáncer. Revista Venezolana de Oncología, 25(2) 190-195.

Galindo V., O., Benjet, C., Juárez G., F., Rojas C., E., Riveros R., A., Aguilar P., J.L. y Alvarado A., S. (2015). Propiedades psicométricas de la Escala Hospitalaria de Ansiedad y Depresión (HADS) en una población de pacientes oncológicos mexicanos. Salud Mental, 38(4), 253-258. doi: 10.17711/SM.0185-3325.2015.035.

Galindo V., O., Rojas C., E., Meneses G., A., Aguilar P., J.L. y Alvarado A., S. (2016). Propiedades psicométricas del Inventario de Depresión de Beck II en pacientes con cáncer. Psicología y Salud, 26(1), 43-49.

Guerrero A., J.M., Prepo S., A.R. y Loyo Á., J.G. (2016). Autotrascendencia, ansiedad y depresión en pacientes con cáncer en tratamiento. Revista Habanera de Ciencias Médicas, 15(2), 297-309.

Hernández, M., Cruzado, J.A., Prado, C., Rodríguez, E., Hernández, C., González, M.A. y Martín, J.C. (2012). Salud mental y malestar emocional en pacientes con cáncer. Psicooncología, 9(2-3), 233-257. doi: 10.5209/rev_PSIC.2013.v9.n2-3.40895.

Hernández, M. y Cruzado, J.A. (2013). La atención psicológica a pacientes con cáncer: de la evaluación al tratamiento. Clínica y Salud, 24(1), 1-9. doi: 10.5093/c12013a1.

Landa R., E., Cárdenas L., G., Greer, J.A., Sánchez R., S. y Riveros R., A. (2014). Evaluación de la depresión en pacientes con cáncer terminal y su aplicación en el contexto mexicano: una revisión. Salud Mental, 37(5), 415-422.

Moreno A., A., Krikorian, A. y Palacio G., C. (2015). Malestar emocional, ansiedad y depresión en pacientes oncológicos colombianos y su relación con la competencia percibida. Avances en Psicología Latinoamericana, 33(3), 517-529. doi: http:// dx.doi.org/10.12804/revistas.urosario.edu.co/apl/a.3148.

Olivan B., B., Rubio A., E., García S., O. y Magallón B., R. (2016). Correlación entre el diagnóstico de depresión y la sintomatología presentada en pacientes de atención primaria. Actas Españolas de Psiquiatría, 44(2), 55-63.

Organización Mundial de la Salud (2014). Perfiles oncológicos de los países. Ginebra: OMS.

Organización Mundial de la Salud (OMS) (2017a). Cáncer. Nota descriptiva. Ginebra: OMS. 
Organización Mundial de la Salud (OMS) (2017b). 10 datos sobre el cáncer. Ginebra: OMS.

Ortiz G., E., Méndez S., L. P., Camargo B., J.A., Chavarro S., A., Toro C., G.I. y Vernaza G., M. B. (2014). Relación entre las estrategias de afrontamiento, ansiedad, depresión y autoestima en un grupo de adultos con diagnóstico de cáncer. Psychologia. Avances de la Disciplina, 8(1) 77-83.

Park, E.M. y Rosenstein, D.L. (2015). Depression in adolescents and young adults with cancer. Dialogues in Clinical Neuroscience, 17(2), 171-180.

Pousa R., V., Miguelez A., A., Hernández B., M., González T., M.A. y Gaviria, M. (2015). Depresión y cáncer: una revisión orientada a la práctica clínica. Revista Colombiana de Cancerología, 19(3), 166-172. doi: 10.1016/j.rccan.2015.04.005.

Prieto, J.M., Blanch, J., Atala, J., Carreras, E., Rovira, M., Cirera, E. y Gastó, C. (2002). Psychiatric morbidity and impact on hospital length of stay among hematologic cancer patients receiving stem-cell transplantation. Journal of Clinical Oncology, 20, 1907-1917.

Sánchez H., M.S., Figueroa L., C.G., Cacho D., B. y Robles G., R. (2016). Relación entre síntomas autonómicos con niveles de ansiedad y depresión en mujeres con cáncer de mama. Claves del Pensamiento Journal, 10(19), 145-162.

Teng, C.T., De Castro, H. y Navas, F.D. (2005). Depressão e comorbidades clínicas. Revista de Psiquiatría Clínica, 32(3), 149-159.

Velásquez S., S. y Zuluaga S., L. (2015). Factores relacionados con la depresión en pacientes pediátricos con cáncer y participación de la enfermera en su detección. Duazary, 12(2), 164.

Zigmond, A.S. y Snaith, R.P. (1983). The Hospital Anxiety and Depression Scale. Acta Psychiatrica Scandinavica, 67(6), 361-370. 